\title{
The effect of ECS on the hippocampus*
}

\author{
JOSEPH B. KEYES \\ Louisiana State Lniversity. Baton Rouge, Louisiana 70803
}

\begin{abstract}
In Experiment I, 64 naive male albino rats were given either ECS or no treatment. The Ss were then tested in a T-maze for spontaneous alternation, a response highly related to hippocampal inactivation. Spontaneous alternation was reduced to near chance level in rats tested 4 or $24 \mathrm{~h}$ after receiving ECS. In a second experiment, rats received ECS or no treatment following the learning of a position habit. Rats were deficient in learning a position habit reversal when trained $4 \mathrm{~h}$ after receiving ECS. The results indicated that ECS seems to produce a temporary inactivation of the hippocampus.
\end{abstract}

The most widely accepted explanation of the effect of electroconvulsive shock (ECS) has been the memory consolidation hypothesis (McGaugh, 1966). This theory suggests that following sensory input, memory is in the form of a trace which undergoes neural consolidation and thereby becomes fixed or permanent. Presumably, then, the massive electrical interference produced by ECS interrupts the consolidation process and prevents the permanent fixation of the trace.

The evidence to support this theory comes primarily from experiments in which a single ECS follows a one-trial passive avoidance task (McGaugh \& Herz, 1972). In a retention test given later, Ss seem to show retrograde amnesia (RA) for the passive avoidance task.

However, a number of studies have shown recovery from ECS-produced RA (Cooper \& Koppenaal. 1964: Kohlenberg \& Trabasso, 1968: Young \& Galluscio, 1971). These studies do not support the memory consolidation hypothesis which predicts that ECS should produce a permanent memory loss. but rather they suggest that ECS produces some temporary effect.

Vanderwolf (1963) demonstrated that rats given ECS reached criterion faster than control animals in a two-way active avoidance task. If ECS produces RA, then two-way active avoidance acquisition should be impaired. not improved. Vanderwolf (1963) suggested that the ECS-produced deficit in passive avoidance performance and improvement in shuttlebox avoidance acquisition mimics the behavioral effects of hippocampal lesions. Isaacson and Wickelgren (1962) have shown passive avoidance deficits as a result of hippocampal lesions. and Isaacson. Douglas, and Moore (1961) showed enhancement of a shuttlebox avoidance with these same lesions.

Olton (1973), in reviewing the effects of brain lesions on avoidance behavior, found that the combination of a decrement in passive avoidance learning and facilitation of a two-way active avoidance task occurs only with hippocampal or septal lesions. Septal lesions, in addition. produce viciousness which is not seen in hippocampal animals (Fried, 1972) or in animals given ECS.

*This research was supported in part by a grant from the Graduate Research Council, LSU, to A. G. Young.
Keyes and Young (1973) and Keyes (1973a) have shown that ECS produces an increase in responding during the extinction of a leverpress task. Keyes and Dempsey (1973) found that with a runway response, ECS administered immediately following the completion of acquisition increased resistance to extinction. These findings are consistent with studies which have found that hippocampal lesions increase resistance to extinction for a leverpress task (Jarrard, 1965) and also for a runway response (Jarrard, Isaacson, \& Wickelgren, 1964).

In addition, Keyes (1973b) has shown that ECS seems to impair performance on a well-trained go, no-go brightness discrimination. The results indicated that ECS interfered with a rat's ability to withhold (inhibit) responding during the no-go stimulus condition. Woodruff, Means, and Isaacson (1973) have found a similar increase in responding during the no-go stimulus condition following hippocampal lesions. These recent findings suggest that ECS may produce response perseveration which is similar to the perseveration explanation of the effect of hippocampal lesions (Kimble. 1968).

Douglas (1967) has noted that the hippocampus and its connections are highly susceptible to seizures. French, Gernandt, and Livingston (1956) have suggested that structures with low seizure thresholds, like the hippocampus, may be depressed by ECS.

The following experiments were therefore conducted to determine if ECS interferes with normal hippocampal functioning.

\section{EXPERIMENT I}

The most sensitive behavior for hippocampal lesions is the phenomenon of spontaneous alternation (Douglas \& Scott. 1972). A normal rat alternates, enters the alley of a T-maze opposite the alley entered on the previous trial, about $85 \%$ of the time. However, hippocampal-lesioned rats alternate only about $50 \%$ of the time (Roberts. Dember. \& Brodwick, 1962). Douglas and Scott (1972) 
Table 1 Mean Percent Alternation

\begin{tabular}{ccccc}
\hline Treatment & $4 \mathrm{~h}$ & $24 \mathrm{~h}$ & $48 \mathrm{~h}$ & $72 \mathrm{~h}$ \\
\hline ECS & 47.50 & 58.75 & 75.00 & 82.50 \\
NT & 83.75 & 82.50 & 83.75 & 86.25 \\
\hline
\end{tabular}

noted that if the hippocampus were inactivated by some treatment, then spontaneous alternation should decrease. Experiment I was conducted to determine whether ECS reduces spontaneous alternation.

\section{Method}

\section{Subjects}

The Ss were 64 naive male albino rats, $175-200 \mathrm{~g}$ in weight at the start of the experiment.

\section{Apparatus}

A wooden T-maze painted flat black was used in testing. The main alley was $38.40 \mathrm{~cm}$ long, and the side alleys were each $38.40 \mathrm{~cm}$ in length. A guillotine door was used between the start box and the main alley and between the choice point and each of the side arms. All the alleys were $11.52 \mathrm{~cm}$ wide and $12.80 \mathrm{~cm}$ high.

\section{Procedure}

The Ss were randomly selected from the LSU colony and placed in individual cages. Food, consisting of Purina Chow, and water were available at all times.

For 5 days, Ss were handled in pairs for $5 \mathrm{~min}$ a day. The Ss were then randomly divided, resulting in the following eight groups $(\mathrm{N}=8)$ :

Groups ECS(4), ECS(24), ECS(48), and ECS(72)-For Ss in these four groups, microalligator clips connected to an ECS source were attached to S's ears. A 50-mA 500-msec ECS was delivered through the earclip electrodes. The Ss were then tested for spontaneous alternation in the T-maze $4,24,48$, or $72 \mathrm{~h}$ after receiving ECS.

Groups NT(4), NT(24), NT(48), and NT(72)-For Ss in these groups, treatment was identical to the four groups above except that no ECS was delivered. The Ss were tested for spontaneous alternation in the T-maze $4,24,48$, or $72 \mathrm{~h}$ after pseudo-ECS.

Testing consisted of 11 nonreinforced trials in the T-maze, thereby allowing for a maximum of 10 alternations. On the first trial, $S$ was placed in the startbox and the door to the main alley was raised. When $S$ had entered (including hindpaws) one of the side alleys, the door was lowered and the response recorded. After $30 \mathrm{sec}, \mathrm{S}$ was removed from the side alley and replaced in the startbox for a second, identical, trial. An alternation was recorded when $S$ went to the opposite side arm as on the preceding trial.

\section{Results}

The percentage of times each $S$ alternated was calculated. The mean percent alternations for the ECS and no treatment groups are shown in Table 1.

A Kruskal-Wallis one-way analysis of variance by ranks for the NT groups was not significant at the .05 level. There was a significant difference among the ECS groups beyond the .01 level (Kruskal-Wallis). A priori group comparisons (Mann-Whitney U tests) showed that ECS(4) vs NT(4), ECS(24) vs NT(24), ECS(4) vs $\operatorname{ECS}(48), \operatorname{ECS}(4)$ vs $\operatorname{ECS}(72)$, and $\operatorname{ECS}(24)$ vs $\operatorname{ECS}(72)$ were significant beyond the .01 level. The comparisons ECS(4) vs ECS(24) and ECS(24) vs ECS(48) were significant beyond the .05 level. The comparisons ECS(48) vs NT(48), ECS(72) vs NT(72), and ECS(48) vs ECS(72) were not significant at the .05 level.

\section{EXPERIMENT II}

Kimble and Kimble (1965) and Thompson and Langer (1963) have shown that hippocampal lesions produce a deficit in learning a reversal of a position habit. Since Experiment I indicated that ECS may temporarily inactivate the hippocampus, this treatment should also impair a position habit reversal.

\section{Method}

\section{Subjects and Apparatus}

The Ss were 64 naive male albino rats, $175-200 \mathrm{~g}$ in weight at the start of the experiment. The apparatus was identical to that used in Experiment I.

\section{Procedure}

The Ss were randomly selected from the LSU colony. They were placed in individual cages and on a food deprivation schedule consisting of $10 \mathrm{~g}$ Purina Chow ever $24 \mathrm{~h}$. Water was available in the cages at all times, and Ss were fed approximately 10 min after each experimental session. From Day 1 to Day 4, Ss were adapted to the deprivation schedule. From Day 5 to Day 8, Ss were handled in pairs for $5 \mathrm{~min}$ daily, and on the 9 th day were allowed to explore the maze for $10 \mathrm{~min}$.

On the 10th day Ss were trained on the initial position habit. The $S$ was placed in the startbox and the door was raised. When $S$ had entered (including hindpaws) one of the side arms, the door was lowered and the response recorded. The $S$ was confined in the side arm for $15 \mathrm{sec}$ following each run, and the intertrial interval spent in a waiting cage was $15 \mathrm{sec}$. One-half the Ss were trained to run to the right side arm and the other Ss to the left side arm of the T-maze. Reinforcement consisted of two .045-g Noyes food pellets. This procedure was continued until $S$ reached a criterion of eight successive correct responses.

The Ss were randomly subdivided, resulting in the following eight groups $(\mathrm{N}=8)$ :

Groups ECS(4), ECS(24), ECS(48), and ECS(72)-For Ss in these four groups, upon reaching criterion, microalligator clips connected to an ECS source were attached to the S's ears. A 50-mA 500-msec ECS was delivered through the earclip electrodes. The Ss were then trained on the reversal task in the T-maze $4,24,48$, or $72 \mathrm{~h}$ after receiving ECS.

Groups NT(4), NT(24), NT(48), and NT(72)-For Ss in these four groups, treatment was identical to the four groups above except that no ECS was delivered. The Ss were trained on the reversal task in the T-maze $4,24,48$, or $72 \mathrm{~h}$ after pseudo-ECS.

Reversal learning consisted of training $S$ to run to the side arm of the T-maze opposite that reinforced in the original training phase. The procedure was identical to that used in original training, and reinforcement again consisted of two $.045 \mathrm{~g}$ - Noyes 
food pellets. The Ss were run to a criterion of eight successive correct responses.

\section{Results}

There was no difference in the number of trials to criterion in the learning of the original position habit for the NT and ECS groups (Mann-Whitney U). Therefore, the number of trials to criterion for the reversal task was analyzed directly.

The results showed that the greatest number of trials to criterion were run by Group ECS(4). followed in order by Groups ECS(24), NT(72), NT(24), ECS(72), ECS(48), NT(4), and NT(48). These data were subjected to an analysis of variance which showed that the main effects of treatment and hour of reversal learning and the Treatment by Hour interaction were all significant beyond the .01 level. A priori group comparisons (t tests) indicated that the comparisons ECS(4) vs NT(4). ECS(4) vs ECS(24), ECS(4) vs ECS(48). and ECS(4) vs ECS(72) were significant beyond the .01 level. The comparisons $\operatorname{ECS}(24)$ vs $\operatorname{ECS}(48)$ and $\operatorname{ECS}(24)$ vs ECS(72) were significant beyond the .05 level. The comparisons ECS(24) vs NT(24). ECS(48) vs NT(48), ECS(72) vs NT(72), and ECS(48) vs ECS(72) were not significant at the .05 level. The mean number of trials to criterion for the reversal task for all groups is shown in Table 2.

\section{GENERAL DISCUSSION}

The results of Experiment I indicate that spontaneous alternation in a T-maze is greatly reduced 4 and $24 \mathrm{~h}$ after ECS. Animals given ECS alternate about $50 \%$ of the time, approximately the same amount as hippocampal-lesioned animals (Roberts. Dember, \& Brodwick. 1962).

Since a reduction in spontaneous alternation indicates an inactivated hippocampus (Douglas \& Scott. 1972). the present findings suggest that ECS may temporarily interfere with normal hippocampal functioning. The inactivation seems to be only temporary. since animals tested at 48 and $72 \mathrm{~h}$ after receiving ECS show a normal amount of alternation.

Kimble and Kimble (1965) and Douglas (1967) suggest that hippocampal lesions produce perseveration of a learned or unlearned prepotent response. The results of Experiment II show that ECS may have a similar effect. Animals given ECS following training on a position habit have a deficit in learning a reversal task when tested $4 \mathrm{~h}$ later. After $24 \mathrm{~h}$, Ss given ECS take longer to learn the reversal task than Ss given no treatment. although the difference is not statistically significant. If ECS produces RA. then Ss given ECS should have less interference from the original position habit and therefore should learn the reversal task faster than control Ss. not slower.
Table 2

Mean Number of Trials to Criterion

\begin{tabular}{ccccc}
\hline Treatment & $4 \mathrm{~h}$ & $24 \mathrm{~h}$ & $48 \mathrm{~h}$ & $72 \mathrm{~h}$ \\
\hline ECS & 35.63 & 28.88 & 23.38 & 23.75 \\
NT & 23.13 & 23.88 & 21.25 & 24.88 \\
\hline
\end{tabular}

These results are consistent with previous findings which showed that ECS produces perseveration for a leverpress task (Keyes, 1973a; Keyes \& Young, 1973) and for a runway response (Keyes \& Dempsey, 1973). The present studies also agree with the finding that ECS impairs an animal's ability to withhold responding during the no-go stimulus condition of a go, no-go discrimination (Keyes, 1973b).

Douglas (1967) and Kimble (1968) have suggested that the hippocampus is involved in an animal's ability to withhold or inhibit prepotent responses. Hippocampal lesions, then, produce perseveration behavior, and therefore tasks which require the animal to inhibit responding are disrupted. The combined ECS studies suggest that ECS also produces perseveration similar to that produced by hippocampal lesions. Electroconvulsive shock may, then, temporarily interfere with normal hippocampal functioning. The present findings also indicate that this inactivation of the hippocampus seems to last for a period of approximately 4 to $24 \mathrm{~h}$.

Routtenberg. Zechmeister, and Benton (1970) have found that the electroencephalogram (EEG) pattern of the hippocampus is abnormal for a period of from 4 to $6 \mathrm{~h}$ following the administration of ECS. Similarily, Landfield, McGaugh, and Tusa (1972) report that following ECS. the amount of theta rhythm $(4-9 \mathrm{~Hz})$ is temporarily decreased. Kety, Javoy, Thierry, Julou, and Glowinski (1967) have shown that norepinephrine and tyrosine hydroxylase levels are elevated for $24 \mathrm{~h}$ after ECS. These studies are consistent with the 4- to 24-h functional inactivation of the hippocampus reported in the present studies.

\section{REFERENCES}

Cooper. R. M., \& Koppenaal, R. J. Suppression and recovery of a one-trial avoidance response after a single ECS. Psychonomic Science, 1964, 1. 303-304.

Douglas, R. J. The hippocampus and behavior. Psychological Bulletin, 1967, 67, 416-442.

Douglas. R. J.. \& Scott, D. W. The differential effects of nitrazepam on certain inhibitory and excitatory behavior. Psychonomic Science. 1972. 26. 164-166.

French. J. D.. Gernandt. B. E.. \& Livingston. R. B. Regional differences in seizure susceptibility in monkey cortex. American Medical Assuciation Archives of Neurology \& Psychiatry. 1956. 75, 260-274.

Fried, P. A. Septum and behavior: A review. Psychological Bulletin. 1972. 78. 292-310.

Isaacson. R. L., \& Wickelgren. W. O. Hippocampal ablation and passive avoidance. Science. 1962. 138. 1104-1106.

Isaacson. R. L.. Douglas. R. J.. \& Moore. R. Y. The effect of 
radical hippocampal ablations on acquisition of avoidance response. Journal of Comparative \& Physiological Psychology, $1961,54,625-628$.

Jarrard, L. E. Hippocampal ablation and operant behavior in the rat. Psychonomic Science, 1965, 2, 115-116.

Jarrard, L. E., Isaacson, R. L.. \& Wickelgren, W. O. Effects of hippocampal ablation and intertrial interval on runway acquisition and extinction. Journal of Comparative \& Physiological Psychology, 1964, 57, 442-444.

Kety, S. S., Javoy, F., Thierry, A. M., Julou, L., \& Glowinski, J. A sustained effect of electroconvulsive shock on the turnover of norepinephrine in the central nervous system of the rat. Proceedings of the National Academy of Science U.S.A., $1967,58,1249-1254$.

Keyes, J. B. ECS perseveration effect following varying amounts of training. Physiological Psychology, 1973a, 1, 2-4.

Keyes, J. B. ECS-produced disruption of a go, no-go discrimination in rats. Bulletin of the Psychonomic Society, $1973 \mathrm{~b}, 1,439-440$.

Keyes, J. B., \& Dempsey, G. L. The effect of motivational state on ECS-induced perseveration. Physiological Psychology, 1973, 1, 133-135.

Keyes, J. B., \& Young, A. G. ECS effects: The PRE. Bulletin of the Psychonomic Society, 1973, 1, 39-40.

Kimble, D. P. The hippocampus and internal inhibition. Psychological Bulletin, 1968, 70, 285-295.

Kimble, D. P., \& Kimble, R. J. Hippocampectomy and response perseveration in the rat. Journal of Comparative \& Physiological Psychology, 1965, 60, 474-476.

Kohlenberg, R., \& Trabasso, T. Recovery of a conditioned emotional response after one or two electroconvulsive shocks. Journal of Comparative \& Physiological Psychology, 1968, 65, 270-273.
Landfield, P. W., McGaugh, J. L., \& Tusa, R. J. Theta rhythm: A temporal correlate of memory storage in the rat. Science, $1972,175,87-89$.

McGaugh, J. L. Time-dependent processes in memory storage. Science, 1966, 153, 1351-1358.

McGaugh, J. L., \& Herz, M. J. Memory consolidation. San Francisco: Albion, 1972.

Olton, D. S. Shock-motivated avoidance and the analysis of behavior. Psychological Bulletin, 1973, 79, 243-251.

Roberts, W. W., Dember, W. N., \& Brodwick, M. Alternation and exploration in rats with hippocampal lesions. Journal of Comparative \& Physiological Psychology, 1962, 55, 695-700. Routtenberg, A., Zechmeister, E. B., \& Benton, C. Hippocampal activity during memory disruption of passive avoidance by electroconvulsive shock. Life Sciences, 1970, 9, 909-918.

Thompson, R., \& Langer, S. K. Deficits in position reversal learning following lesions of the limbic system. Journal of Comparative \& Physiological Psychology, 1963, 56, 987-995.

Vanderwolf, C. H. Improved shuttle-box performance following electroconvulsive shock. Journal of Comparative \& Physiological Psychology, 1963, 56, 983-986.

Woodruff, M. L., Means, L. W., \& Isaacson, R. L. Deficient go, no-go brightness discriminations in rats following hippocampal lesions. Physiological Psychology, 1973, 1, 85-88.

Young, A. G., \& Galluscio, E. H. Recovery from ECS-produced amnesia. Psychonomic Science, 1971, 22, 149-151.

(Received for publication June 6, 1973; revision received September 6, 1973; accepted September 10, 1973.) 